\title{
New Developments in Cosmology and Gravitation from Extended Theories of General Relativity
}

\author{
Mauricio Bellini, ${ }^{1}$ Kishor Adhav, ${ }^{2}$ José Edgar Madriz Aguilar, ${ }^{3}$ and Dandala R. K. Reddy \\ ${ }^{1}$ IFIMAR, University of Mar del Plata and CONICET, Funes 3350, 7600 Mar del Plata, Argentina \\ ${ }^{2}$ Department of Mathematics, Sant Gadge Baba Amravati University, Amaravati, Mahasastra 444602, India \\ ${ }^{3}$ Department of Mathematics, CUCEI, University of Guadalajara, Avenue Revolución 1500 S. R, 44430 Guadalajara, Mexico \\ ${ }^{4}$ Department of Science and Humanities, MVGR College of Engineering, Vizianagaram, Andhra Pradesh 530017, India
}

Correspondence should be addressed to Mauricio Bellini; mbellini@mdp.edu.ar

Received 29 January 2014; Accepted 29 January 2014; Published 3 March 2014

Copyright (C) 2014 Mauricio Bellini et al. This is an open access article distributed under the Creative Commons Attribution License, which permits unrestricted use, distribution, and reproduction in any medium, provided the original work is properly cited. The publication of this article was funded by SCOAP S $^{3}$

Extensions and modifications to the standard 4D theory of general relativity are topics that have an increasing impact in top original research on gravitation and cosmology. This issue compiles exciting papers that can be very interesting for researchers who could be interested in the study of extended theories of general relativity focused on cosmology and gravitation.

An important topic for the present day cosmology consists in explaining why the universe is accelerated and how this acceleration is related to an unknown kind of energy called "dark energy." In this issue it is considered a cosmic fluid as a quasi-static thermodynamic system. The status of the generalized second law of thermodynamics is investigated and the range of validity for the equation of state parameter is derived for a few important cosmological models. The physical origin of dark energy can be explored using a condensate of spinors which are free of interactions in a 5D relativistic vacuum defined in an extended de Sitter spacetime which is Riemann flat. This condensate of spinors could be an interesting candidate to explain the presence of dark energy in the early universe, during the inflationary stage.

A new method for the study of general higher dimensional Kaluza-Klein theories based on the Riemannian adapted connection and on a theory of adapted tensor fields in the ambient space is also interesting to be studied. This topic can be explored in a covariant form and it is very interesting to study the motion on a 4D space induced from a $(4+n) \mathrm{D}$ general gauge Kaluza-Klein space.

Anisotropic cosmological models are really an interesting topic for cosmologists and researchers, especially when this topic is studied in the framework of a scalar-tensor theory of gravitation with a source such that the energy momentum tensor is a bulk viscous fluid containing one-dimensional cosmic strings.

Braneworld cosmology from modified induced gravity on the brane is a very close approach to modern Kaluza-Klein (or Induced Matter theory of gravity). These kinds of approaches can predict very plausible cosmological models that describe the history of the universe.

There are very interesting approaches related to extended theories of gravity applied to topological defects such as black holes. In this context some thermodynamical considerations can be explored when the horizon structure is invariant.

By compiling these papers, we hope to enrich readers with new investigations on this exciting research area and stimulate new investigations which could be fundamental for the future of the physics.

Mauricio Bellini Kishor Adhav José Edgar Madriz Aguilar Dandala R. K. Reddy 

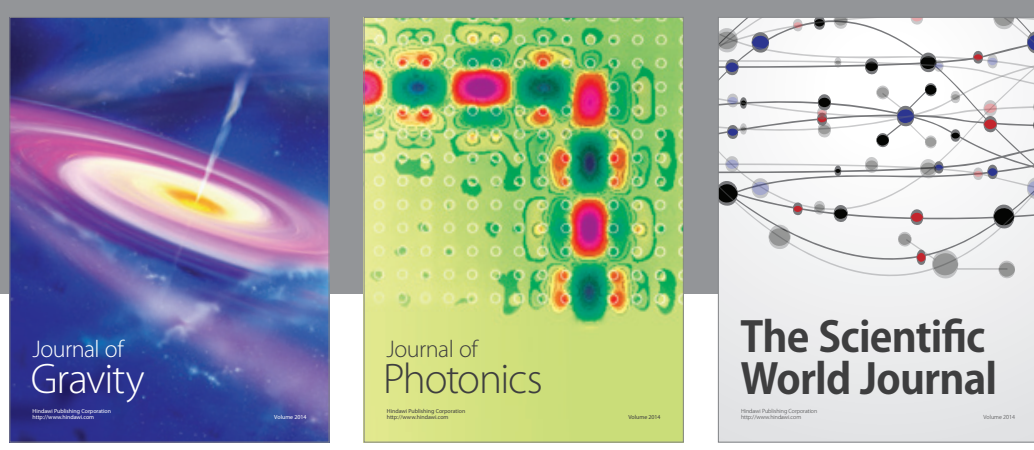

The Scientific World Journal
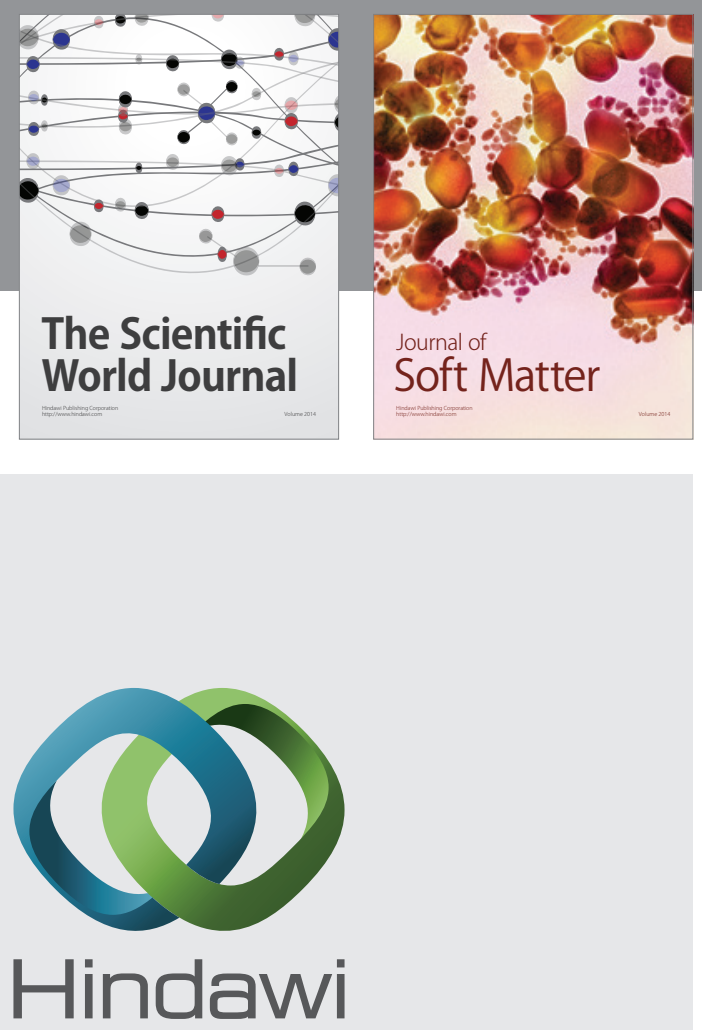

Submit your manuscripts at

http://www.hindawi.com

nternational Journal of

Statistical Mechanics
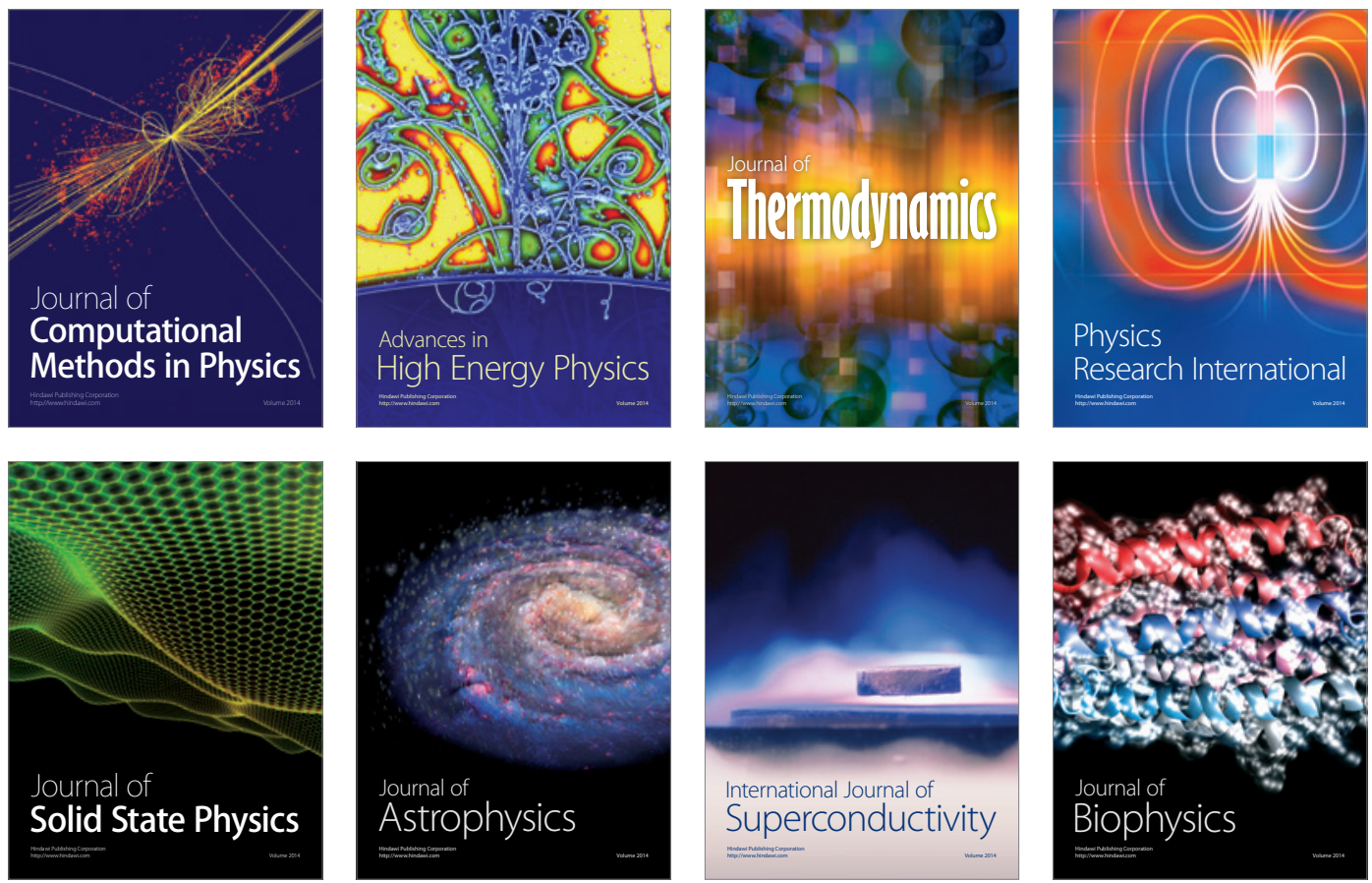
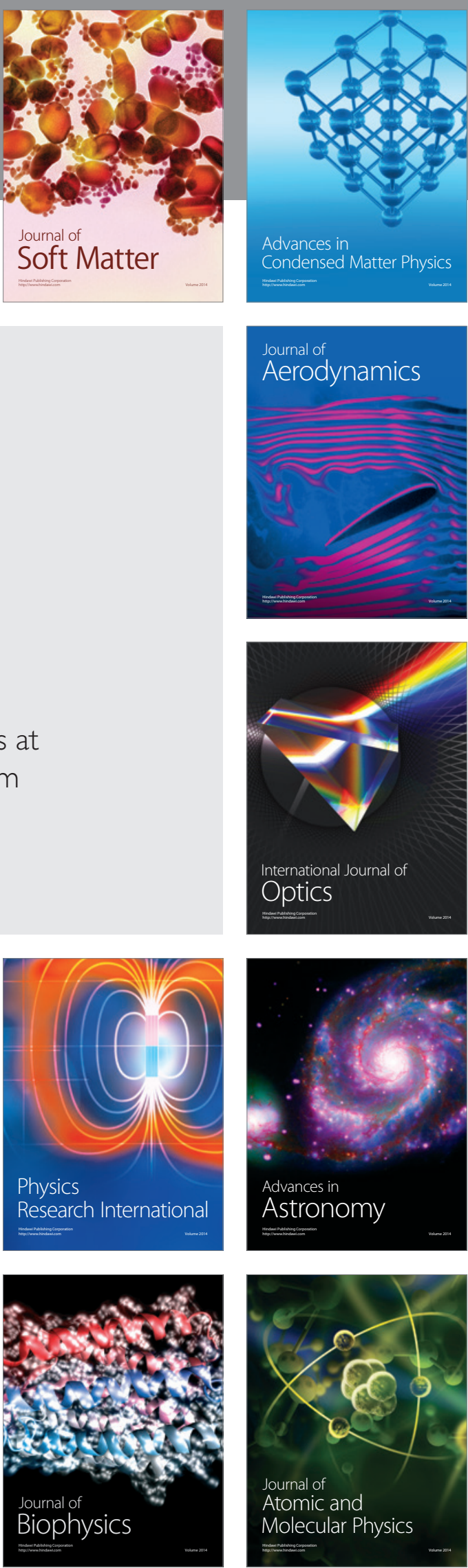